\title{
EFFECTS OF BLACK PEPPER AND SODIUM ASCORBATE ON VOLATILE COMPOUNDS OF SUCUK
}

\author{
Selen Sallan ${ }^{1 *}$, Güzin Kaban ${ }^{2}$, Mükerrem Kaya ${ }^{2}$ \\ ${ }^{1}$ Bandırma Onyedi Eylul University, Bandırma Vocational School, Department of Food Processing, Bandırma, \\ Balıkesir, Turkey \\ ${ }^{2}$ Atatürk University, Faculty of Agriculture, Department of Food Engineering, Erzurum, Turkey \\ Received / Geliş: 30.06.2021; Accepted / Kabul: 18.10.2021; Published online / Online bask1: 27.10.2021 \\ Sallan, S., Kaban, G., Kaya, M. (2021). Effects of black pepper and sodum ascorbate on volatıle compounds of sucuk. \\ GIDA (2021) 46 (6) 1358-1368 doi: 10.15237/gida.GD21107. \\ Sallan, S., Kaban, G., Kaya, M. (2021). Karabiber ve sodyum askorbatın sucuğun uçun bileşikleri üzerine etkileri. \\ GIDA (2021) 46 (6) 1358-1368 doi: 10.15237/gida.GD21107.
}

\begin{abstract}
The effects of different levels of black pepper $(5,10$ or $15 \mathrm{~g} / \mathrm{kg}$ ) and the use of ascorbate (without ascorbate and with ascorbate) on the volatile compounds of sucuk were examined in the study. The volatile compounds of the samples belonging to each group were extracted by solid phase micro-extraction (SPME) and analyzed by gas chromatography-mass spectrometry (GC-MS). Ascorbate was not effective on aldehydes, aromatic hydrocarbons, esters, acids and terpenes. While high black pepper level (15 g/kg) decreased the abundance of hexanal, it increased the ethanol abundance. $\alpha$-pinene and 3-carene increased as black pepper levels increased. Among the terpenes, D-limonene and caryophyllene were the most abundant compounds and were significantly affected by the black pepper level of $15 \mathrm{~g} / \mathrm{kg}$. D-limonene and caryophyllene were the most related compounds for PC1; while allyl mercaptane, $\gamma$-terpinene, D-limonene, hexane for PC2.
\end{abstract}

Keywords: Volatile compound, black pepper, ascorbate, fermented sausage, sucuk, principal component analysis (PCA)

\section{KARABİBER VE SODYUM ASKORBATIN SUCUĞUN UÇUCU BİLEŞİKLERİ ÜZERİNE ETKILLERİ}

\section{ÖZ}

Araştırmada, farklı seviyelerde karabiber (5, 10 veya $15 \mathrm{~g} / \mathrm{kg}$ ) ve askorbat (askorbatsız ve askorbatlı) kullanımının sucuğun uçucu bileşikleri üzerindeki etkileri incelenmiştir. Her gruba ait numunelerin uçucu bileşikleri katı faz mikro ekstraksiyon (SPME) ile ekstrakte edilmiş ve gaz kromatografi-kütle spektrometrisi (GC-MS) ile analiz edilmiştir. Askorbat, aldehitler, aromatik hidrokarbonlar, esterler, asitler ve terpenler üzerinde etkili olmamıştır. Yüksek karabiber düzeyi $(15 \mathrm{~g} / \mathrm{kg}) \mathrm{hekzanal} \mathrm{seviyesini}$ azaltırken, etanol seviyesini artırmıştır. Karabiber seviyesi arttıkça $\alpha$-pinen ve 3-caren artmıştır. Terpenler içerisinde D-limonen ve karyofillen en fazla bulunan bileşikler olup $15 \mathrm{~g} / \mathrm{kg} \mathrm{karabiber}$ seviyesinden önemli ölçüde etkilenmiştir. D-limonen ve karyofillen PC1, allil merkaptan, $\gamma$-terpinen, D-limonen ve hekzan ise PC2 için en ilgili bileşiklerdir.

Anahtar Kelimeler: Uçucu bileşik, karabiber, askorbat, fermente sosis, sucuk, temel bileşen analizi (PCA)

\footnotetext{
* Corresponding author / Yazışmalardan sorumlu yazar

(t): ssallan@bandirma.edu.tr

(ग): (+90) 2666067529
}

县: (+90) 2667149304

Selen Sallan; ORCID no: 0000-0001-9806-6937

Güzin Kaban; ORCID no: 0000-0001-6720-7231

Mükerrem Kaya; ORCID no: 0000-0001-6340-828X 


\section{INTRODUCTION}

Flavor is one of the most important quality attributes for meat products and contributes the acceptability of the product by consumer. The biochemical changes during ripening of fermented sausages determine the taste and aroma of the final product. Carbohydrate fermentation, lipolysis, proteolysis, lipid oxidation and amino acid catabolism are important phenomena with regard to the characteristic flavor of dry and semi-dry fermented sausages such as sucuk, salami, Rohwurst, Chorizo, pepperoni (Ordonez et al., 1999; Kaban, 2010; Kaya and Kaban, 2019). In these reactions, external factors such as the initial fermentation temperature, as well as internal factors such as salt, curing agent, sugar and spices, which are involved in the formulation, have become effective. The abundance and balance of volatile and non-volatile compounds formed as a result of the reactions vary depending on the type of product, and thus different products can be obtained in terms of flavor (Kaya and Kaban, 2019). Volatile compounds contribute to the aroma of the product, while non-volatile compounds such as peptides and free amino acids directly affect the flavor of the product (Toldra, 1998).

The spices used in fermented sausages also contribute to the characteristic flavor and aroma of the product. In the formulation, spices are used in proportions varying between $0.5-2 \%$ depending on the type of product, and even some products are characterized by the specific spices they contain (Ordonez et al., 1999). Black pepper takes place an important position among the spices and it is called "the king of the spices" (Srinivasan, 2007; Zhang et al., 2021). Black pepper is widely used in fresh meat and processed meat products because of its pungent and characteristic taste (Vignolo et al., 2010). Black pepper is also an important spice for sucuk, a type of dry fermented sausage. However, there is no study of the effect of black pepper on sucuk's volatile profile.

The additives also play an important role on flavor of fermented sausages. Salt and nitrite are essential additives for these products. Besides, ascorbate can be also added to the formulation. However, the number of studies on the effect of this reducing compound on the volatile compound profile of fermented sausages is rather limited (Stahnke, 1999). The relationship between the volatile compound profile and ascorbate in sucuk has not yet been investigated (Kaban and Kaya, 2009; Ekici et al., 2015; Yalınkilıç et al., 2015). Therefore, it is aimed to determine the effects of different levels of black pepper $(5 \mathrm{~g} / \mathrm{kg}$, $10 \mathrm{~g} / \mathrm{kg}$ or $15 \mathrm{~g} / \mathrm{kg}$ ) and the use of ascorbate (with or without sodium ascorbate) on the volatile compound profile of sucuk, a type of dry fermented sausage.

\section{MATERIAL AND METHOD}

\section{Material}

Beef meat (round) and beef fat were used as a raw material obtained from two carcasses $(24 \mathrm{~h}$ postmortem). After raw meat was trimmed of visible fat and connective tissue, it was cut into small pieces. Similarly, beef fat was also cut into small pieces. Then, after vacuum packaging, they were separately stored at $-18{ }^{\circ} \mathrm{C}$ for a week.

Autochthonous strains (Lactobacillus plantarum GM77 + Staphylococcus xylosus GM92) were used as starter culture (Kaban and Kaya, 2009). L. plantarum GM77 and S. xylosus GM92 were grown in MRS (Merck, Darmstadt, Germany) and TSB (Merck, Darmstadt, Germany) broth at $30^{\circ} \mathrm{C}$ for 24 h. L. plantarum GM77 was inoculated with $10^{7}$ cfu/g, while S. xylosus GM92 was inoculated with $10^{6} \mathrm{cfu} / \mathrm{g}$ into sucuk batters.

\section{Sucuk Production}

For the sucuk production, $20 \mathrm{~g}$ salt, $10 \mathrm{~g}$ garlic, 4 $\mathrm{g}$ sucrose, $7 \mathrm{~g}$ red pepper, $9 \mathrm{~g}$ cumin, $2.5 \mathrm{~g}$ allspice, $0.15 \mathrm{~g}$ sodium nitrite per $1 \mathrm{~kg}$ beef meat and beef fat were used. Considering this basic formulation, 6 sucuk batters (with starter culture) were made using different levels of black pepper $(5,10$ or 15 $\mathrm{g} / \mathrm{kg}$ ) and sodium ascorbate (0 or $568 \mathrm{mg} / \mathrm{kg}$ ). Five $\mathrm{kg}$ of sucuk batter per each treatment was prepared in laboratory-type bowl cutter (MADO Typ MTK 662, Dornhan / Schwarzwald). For each treatment, two batches were prepared, thus a total of 12 sucuk batches was obtained. 
The sucuk batters were stuffed into collagen casings (Naturin Darm, $38 \mathrm{~mm}$ ) at $200 \pm 10 \mathrm{~g}$ using a laboratory-type filling machine (MADO Typ MTK 591, Dornhan / Schwarzwald). The sausages were ripened in an automatic climate unit (Reich, Germany), where temperature, relative humidity and air flow can be controlled automatically. The ripening program was carried out in the automatic climate unit for 2 days with a temperature of $22 \pm 1^{\circ} \mathrm{C}$, relative humidity of 90 $\pm 2 \%$, and on days $3^{\text {rd }}$ and $4^{\text {th }}$ with a temperature of $20 \pm 1{ }^{\circ} \mathrm{C}$, relative humidity of $88 \pm 2 \%$. On the following days ( $5^{\text {th }}, 6^{\text {th }}$ and $7^{\text {th }}$ days), the temperature was kept constant at $18 \pm 1{ }^{\circ} \mathrm{C}$, and the relative humidity was gradually reduced to 80 $\pm 2 \%$. Air flow was kept at $0.5 \mathrm{~m} / \mathrm{s}$ in the first 3 days of ripening, and gradually decreased to 0.1 $\mathrm{m} / \mathrm{s}$ in the following days (Kaban and Kaya, 2009).

\section{Method}

After sucuk production, two random samples were taken from each treatment in each production and they were subjected to $\mathrm{pH}, \mathrm{a}_{\mathrm{w}}$ and volatile compounds analyzes.

\section{Determination of $\mathrm{pH}$ and $\mathrm{a}_{\mathrm{w}}$}

To determinate to $\mathrm{pH}$ value of samples, $10 \mathrm{~g}$ of sucuk sample was homogenised with $100 \mathrm{~mL}$ distilled water using Ultra-Turrax (T25; IKAWerke GmbH, Staufen, Germany), then it was measured using a pH meter (Orion 420, Thermo, Waltham, MA).

The water acitivity $\left(a_{\mathrm{w}}\right)$ value of samples was determined at $25{ }^{\circ} \mathrm{C}$ using an $a_{\mathrm{w}}$ sprint device (Model TH-500; Novasina AG, Lachen, Switzerland). Before the analysis, the instrument was calibrated with 6 different salt solutions.

\section{Analysis of Volatile Compounds}

Five $g$ each of the homogenized samples were weighed into $40 \mathrm{~mL}$ vials (Supelco, Bellefonte PA, USA). The vials were kept in a thermal block (Supelco, Bellefonte PA, USA) for one hour at 30 ${ }^{\circ} \mathrm{C}$ for the extraction of volatile compounds and it was ensured that volatile compounds were accumulated in the headspace. Then, CAR/PDMS fiber (Supelco, $75 \mu \mathrm{m}$, USA) was placed in vials for adsorption of the compounds and kept for 2 hours. After this step, the fiber was injected into the gas chromatography (GC, Agilent Technologies 6890N) device and the compounds were identified by mass spectrometry (MS, Agilent Technologies 5973). Carrier gas used in the system was helium and DB-624 (60 m x $0.250 \mathrm{~mm} \times 1.40 \mu \mathrm{m}$, Agilent Tech, USA) was used as column. The oven temperature of the gas chromatography was initially set at $40{ }^{\circ} \mathrm{C}$ for 5 $\mathrm{min}$, and then gradually increased to $110^{\circ} \mathrm{C}$ with $3{ }^{\circ} \mathrm{C} / \mathrm{min}, 150^{\circ} \mathrm{C}$ with $4{ }^{\circ} \mathrm{C} / \mathrm{min}$ and $210^{\circ} \mathrm{C}$ with $10{ }^{\circ} \mathrm{C} / \mathrm{min}$, then kept at $210{ }^{\circ} \mathrm{C}$ for $12 \mathrm{~min}$. The library of mass spectrometry (NIST, WILEY and FLAVOR) and standard mix (Supelco 44585-U, Bellefonte PA USA) were used for identification (Kaban, 2009).

\section{Statistical Analysis}

The use of sodium ascorbate (without or with sodium ascorbate) and black pepper level (5, 10, $15 \mathrm{~g} / \mathrm{kg}$ ) were evaluated as factors. For each treatment, two batters were prepared. The experiments were performed according to the randomised complete block design with two blocks (two replicates). The data was analyzed with two-way ANOVA using the general linear model. The factors were evaluated as fixed effects and replicates as random effect. The means of significant sources of variation were compared using Duncan's multiple comparison test at the $P$ $<0.05$ level. All statistical analyzes were carried out using the SPSS version 24 statistical program (SPSS Inc., Chicago, IL, USA). Principal component analysis (PCA) was performed with the aid of The Unscrambler software (CAMO software version 10.1).

\section{RESULTS AND DISCUSSION $\mathrm{pH}$ and $\mathrm{a}_{\mathrm{w}}$}

The overall effect of sodium ascorbate addition and black pepper level on the $\mathrm{pH}$ and $a_{\mathrm{w}}$ of sucuk samples is given Table 1. According to the results, the black pepper level and sodium ascorbate addition did not significantly affect the $\mathrm{pH}$ and $\mathrm{a}_{\mathrm{w}}$ values of the samples $(P>0.05)$. In this study, starter culture was used in all treatments. Both $a_{w}$ and $\mathrm{pH}$ are hurdle effects for fermented sausages. In sucuk samples, $\mathrm{pH}$ and $\mathrm{a}_{\mathrm{w}}$ values were below 
5.0 and 0.90 , respectively (Table 1 ). Similar results have been reported in previous studies on sucuk made with starter culture (Kaban and Kaya, 2009; Sallan et al., 2019).

Table 1: Mean $\mathrm{pH}$ and $\mathrm{a}_{\mathrm{w}}$ values for sucuk samples (Mean $\pm \mathrm{SD}$ )

\begin{tabular}{|c|c|c|c|}
\hline Treatment & $\mathrm{n}$ & $\mathrm{pH}$ & $a_{w}$ \\
\hline $\begin{array}{l}\text { Sodium Ascorbate } \\
(\text { Asc) }\end{array}$ & & & \\
\hline without Asc & 12 & $4.86 \pm 0.06$ & $0.87 \pm 0.04$ \\
\hline with Asc & 12 & $4.89 \pm 0.05$ & $0.89 \pm 0.02$ \\
\hline Significance & & $\mathrm{ns}$ & $\mathrm{ns}$ \\
\hline $\begin{array}{l}\text { Black Pepper Level } \\
\text { (BP) }\end{array}$ & & & \\
\hline $5 \mathrm{~g} / \mathrm{kg}$ & 8 & $4.90 \pm 0.03$ & $0.88 \pm 0.01$ \\
\hline $10 \mathrm{~g} / \mathrm{kg}$ & 8 & $4.86 \pm 0.06$ & $0.89 \pm 0.02$ \\
\hline $15 \mathrm{~g} / \mathrm{kg}$ & 8 & $4.86 \pm 0.08$ & $0.87 \pm 0.05$ \\
\hline Significance & & ns & $\mathrm{ns}$ \\
\hline Asc $\times$ BP & & ns & ns \\
\hline
\end{tabular}

ns: not significant, $P>0.05$; $\mathrm{SD}$ : standard deviation.

\section{Volatile Compounds}

The overall effects of black pepper level and sodium ascorbate addition on the volatile compound profile of sucuk are given in Table 2. A total of 47 compounds, including 5 aldehydes, 4 aliphatic hydrocarbons, 1 alcohol, 2 aromatic hydrocarbons, 1 acid, 2 ester, 8 sulfur compounds and 24 terpene compounds, were identified in the sucuk samples (Table 2).

Aroma is one of the most important quality attributes in fermented sausages such as sucuk. Compounds formed by biochemical reactions such as lipolysis, proteolysis, lipid oxidation, amino acid catabolism, strecker degradation during fermentation and drying contribute to the aroma (Kaban, 2010; Xiao et al., 2020). The spices in the formulation can also contribute more or less effectively to the aroma (Ordonez et al., 1999).

The use of sodium ascorbate showed no significant effect on aldehydes $(P>0.05)$, while the black pepper reduced the abundance of hexanal and 2-methyl-3 phenyl propanal in sucuk $(P<0.05)$. In fermented sausages, aldehydes can be formed through strecker degradation, carbohydrate metabolism or lipid oxidation (Ordonez et al., 1999). The fact that high black pepper levels $(15 \mathrm{~g} / \mathrm{kg})$ decrease both hexanal and 2-methyl-3 phenyl propanal levels in sucuk samples in the study may be due to black pepper being a good source of flavonoid and phenolic antioxidants (Zarai et al., 2013). Hexanal is an indicator of lipid oxidation. This compound possesses the typical smell of green grass (Sidira et al., 2015). 2-methyl-3 phenyl propanal is the characteristic aldehyde for sucuk (Kaban, 2010). As in the present study, Stahnke (1999) reported that ascorbate had no effect on aldehydes.

Black pepper had no effect on aliphatic hydrocarbons $(P>0.05)$. Sodium ascorbate, on the other hand, was effective only on hexane $(P$ $<0.05)$. Due to the high threshold values of aliphatic hydrocarbons, their effects on the aroma of fermented sausages are very limited (Ruiz et al., 1999; Ramirez and Cava, 2007). Hexane also had the highest abundance among aliphatic hydrocarbons (Table 2).

Black pepper level did not significantly affect the aromatic hydrocarbons $(P>0.05)$. Similarly, sodium ascorbate had no significant effect on these compounds $\left(\begin{array}{ll}P & >0.05)\end{array}\right.$ (Table 2). In addition, 2 aromatic hydrocarbons were determined in sucuk samples. These compounds were previously determined in a study on sucuk conducted by Kaban (2010).

Ethyl alcohol was the only alcohol that was determined in sucuk samples. The use of ascorbate decreased the level of this compound, while $15 \mathrm{~g} / \mathrm{kg}$ black pepper level increased ethanol formation $(P<0.05)$. However, there was no statistically significant difference between addition of $5 \mathrm{~g} / \mathrm{kg}$ and $10 \mathrm{~g} / \mathrm{kg}(P>0.05)$ (Table 2). Alcohols are generated as reaction products of lipid oxidation, carbohydrate metabolism and amino acid catabolism in dry fermented sausages (Mateo and Zumalacarregui, 1996; Ordonez et al., 1999). Ethyl alcohol determined in sucuk samples was found to be lower in the presence of ascorbate (Table 2). On the other hand, Sallan et al. (2022) reported that the use of ascorbate in a semi-dry fermented sausage has no effect on ethyl alcohol. In a study carried out by Stahnke (1999), it was reported that the addition of ascorbate significantly increases ethanol level. On the other 
hand, increasing the black pepper level to $15 \mathrm{~g} / \mathrm{kg}$ increased the ethanol level (Table 2). It is thought that these results are related to the redox potential of the meat fermentation (Huynh et al., 2020). Ordonez et al. (1999) also emphasized that alcohol formation is favored by a greater $\mathrm{NADH}$ compared to $\mathrm{NAD}^{+}$concentration.

Only acetic acid was determined as acid in sucuk samples (Table 2). Acetic acid is formed through amino acid catabolism and lipid oxidation as well as activities of homofermantative lactic acid bacteria and staphylococci (Montel et al., 1998). This compound, which gives sour note to the product (Montel et al., 1998), was also identified in previous studies on sucuk (Kaban 2010, Demirok Soncu et al., 2020). However, in our study, neither the black pepper content nor the addition of sodium ascorbate significantly affected the acids $(P>0.05)$ (Table 2$)$.

Table 2. Overall effects of ascorbate addition and black pepper levels on the volatile profile of sucuk (means $\pm \mathrm{SD})\left(\mathrm{AU} \times 10^{\circ}\right)$

\begin{tabular}{|c|c|c|c|c|c|c|c|c|c|}
\hline \multirow[b]{2}{*}{ Compounds } & \multirow[b]{2}{*}{$\mathrm{KI}$} & \multirow[b]{2}{*}{$\mathrm{RT}$} & \multicolumn{3}{|c|}{ Ascorbate Addition (Asc) } & \multicolumn{2}{|c|}{ Black Pepper Level (BP) } & \multicolumn{2}{|c|}{$P$} \\
\hline & & & $\begin{array}{c}\text { Without } \\
\text { Asc }\end{array}$ & With Asc & $5 \mathrm{~g} / \mathrm{kg}$ & $10 \mathrm{~g} / \mathrm{kg}$ & $15 \mathrm{~g} / \mathrm{kg}$ & Asc & $\mathrm{BP}$ \\
\hline \multicolumn{10}{|l|}{ Aldehydes } \\
\hline Pentanal & 742 & A & $0.38 \pm 0.26 \mathrm{a}$ & $0.37 \pm 0.34 \mathrm{a}$ & $0.52 \pm 0.32 \mathrm{a}$ & $0.24 \pm 0.29 \mathrm{a}$ & $0.35 \pm 0.23 \mathrm{a}$ & ns & ns \\
\hline Hexanal & 849 & A & $1.38 \pm 0.86 \mathrm{a}$ & $0.94 \pm 0.84 \mathrm{a}$ & $1.79 \pm 0.91 \mathrm{a}$ & $1.04 \pm 0.89 \mathrm{ab}$ & $0.65 \pm 0.25 b$ & ns & $*$ \\
\hline Octanal & 1051 & A & $0.49 \pm 0.25 \mathrm{a}$ & $1.01 \pm 1.36 \mathrm{a}$ & $1.34 \pm 1.58 \mathrm{a}$ & $0.47 \pm 0.27 \mathrm{a}$ & $0.43 \pm 0.15 \mathrm{a}$ & ns & ns \\
\hline Nonanal & 1143 & A & $2.52 \pm 1.69 \mathrm{a}$ & $4.30 \pm 6.06 \mathrm{a}$ & $5.77 \pm 7.14 \mathrm{a}$ & $2.18 \pm 1.41 \mathrm{a}$ & $2.27 \pm 1.53 \mathrm{a}$ & ns & ns \\
\hline 2-methyl-3-phenyl-propanal & 1344 & $\mathrm{~B}$ & $9.76 \pm 2.49 \mathrm{a}$ & $10.5 \pm 3.16 \mathrm{a}$ & $11.9 \pm 3.15 \mathrm{a}$ & $10.3 \pm 2.44 \mathrm{ab}$ & $8.23 \pm 1.56 \mathrm{~b}$ & ns & $*$ \\
\hline \multicolumn{10}{|l|}{ Aliphatic Hydrocarbons } \\
\hline Hexane & 600 & A & $18.2 \pm 8.67 \mathrm{a}$ & $11.3 \pm 3.75 b$ & $16.6 \pm 9.26 \mathrm{a}$ & $13.70 \pm 7.09 \mathrm{a}$ & $13.93 \pm 6.26 \mathrm{a}$ & $*$ & ns \\
\hline Undecane & 1100 & A & $0.56 \pm 0.38 \mathrm{a}$ & $0.62 \pm 0.45 \mathrm{a}$ & $0.72 \pm 0.49 \mathrm{a}$ & $0.58 \pm 0.50 \mathrm{a}$ & $0.47 \pm 0.08 \mathrm{a}$ & ns & ns \\
\hline Dodecane & 1200 & A & $2.33 \pm 1.44 \mathrm{a}$ & $1.75 \pm 0.79 \mathrm{a}$ & $1.94 \pm 0.90 \mathrm{a}$ & $2.27 \pm 1.22 \mathrm{a}$ & $1.92 \pm 1.44 \mathrm{a}$ & ns & ns \\
\hline Tetradecane & 1400 & A & $2.19 \pm 2.33 \mathrm{a}$ & $1.58 \pm 1.57 \mathrm{a}$ & $1.93 \pm 1.87 \mathrm{a}$ & $2.06 \pm 1.61 \mathrm{a}$ & $1.66 \pm 2.27 \mathrm{a}$ & ns & ns \\
\hline \multicolumn{10}{|l|}{ Alcohol } \\
\hline Ethanol & 505 & A & $0.82 \pm 0.65 a$ & $0.39 \pm 0.31 \mathrm{~b}$ & $0.46 \pm 0.23 b$ & $0.39 \pm 0.34 \mathrm{~b}$ & $0.96 \pm 0.76 \mathrm{a}$ & $*$ & $*$ \\
\hline \multicolumn{10}{|l|}{ Aromatic Hydrocarbons } \\
\hline Styrene & 935 & B & $0.16 \pm 0.22 \mathrm{a}$ & $0.15 \pm 0.13 \mathrm{a}$ & $0.22 \pm 0.22 \mathrm{a}$ & $0.15 \pm 0.18 \mathrm{a}$ & $0.09 \pm 0.09 \mathrm{a}$ & ns & ns \\
\hline $\begin{array}{l}\text { 1,2-dimethoxy-4-(2- } \\
\text { propenyl)- benzene }\end{array}$ & 1434 & $\mathrm{C}$ & $4.94 \pm 1.41 \mathrm{a}$ & $5.65 \pm 1.51 \mathrm{a}$ & $4.79 \pm 1.45 \mathrm{a}$ & $5.65 \pm 1.65 \mathrm{a}$ & $5.45 \pm 1.37 \mathrm{a}$ & ns & ns \\
\hline \multicolumn{10}{|l|}{ Acid } \\
\hline Acetic acid & 710 & A & $6.46 \pm 2.30 \mathrm{a}$ & $6.81 \pm 3.73 \mathrm{a}$ & $7.67 \pm 3.55 \mathrm{a}$ & $5.56 \pm 2.53 \mathrm{a}$ & $6.68 \pm 2.94 \mathrm{a}$ & ns & ns \\
\hline \multicolumn{10}{|l|}{ Esters } \\
\hline Methyl acetate & 890 & $\mathrm{C}$ & $1.01 \pm 0.43 \mathrm{a}$ & $0.69 \pm 0.44 \mathrm{a}$ & $1.09 \pm 0.51 \mathrm{a}$ & $0.72 \pm 0.43 \mathrm{a}$ & $0.74 \pm 0.39 \mathrm{a}$ & ns & ns \\
\hline Hexyl butyrate & 1215 & B & $1.43 \pm 1.03 \mathrm{a}$ & $1.00 \pm 0.50 \mathrm{a}$ & $1.55 \pm 0.96 \mathrm{a}$ & $1.41 \pm 0.85 \mathrm{ab}$ & $0.68 \pm 0.28 \mathrm{~b}$ & ns & $*$ \\
\hline \multicolumn{10}{|l|}{ Sulfur Compounds } \\
\hline Allyl mercaptan & 574 & B & $18.6 \pm 8.60 \mathrm{a}$ & $13.7 \pm 6.99 \mathrm{a}$ & $19.43 \pm 9.3 \mathrm{a}$ & $12.26 \pm 5.47 \mathrm{a}$ & $16.62 \pm 8.18 \mathrm{a}$ & ns & ns \\
\hline Allyl methyl sulfide & 730 & $\mathrm{~B}$ & $4.21 \pm 2.45 \mathrm{a}$ & $2.33 \pm 1.56 \mathrm{~b}$ & $4.39 \pm 2.89 \mathrm{a}$ & $2.54 \pm 1.74 \mathrm{a}$ & $2.87 \pm 1.64 \mathrm{a}$ & $*$ & ns \\
\hline 1-(methylthio)-1-propene & 832 & $\mathrm{C}$ & $0.36 \pm 0.20 \mathrm{a}$ & $0.36 \pm 0.26 \mathrm{a}$ & $0.52 \pm 0.21 \mathrm{a}$ & $0.19 \pm 0.18 \mathrm{~b}$ & $0.36 \pm 0.18 \mathrm{ab}$ & ns & $*$ \\
\hline Dimethyl disulfide & 834 & B & $0.98 \pm 0.43 \mathrm{a}$ & $0.71 \pm 0.37 \mathrm{a}$ & $1.15 \pm 0.45 \mathrm{a}$ & $0.71 \pm 0.38 b$ & $0.67 \pm 0.23 \mathrm{~b}$ & ns & $*$ \\
\hline 3,3'-thiobis-1-propene & 888 & $\mathrm{~B}$ & $3.01 \pm 1.88 \mathrm{a}$ & $1.25 \pm 0.77 \mathrm{~b}$ & $2.96 \pm 2.05 \mathrm{a}$ & $1.78 \pm 1.72 \mathrm{a}$ & $1.63 \pm 0.87 \mathrm{a}$ & $* *$ & ns \\
\hline
\end{tabular}


Table 2. continue

\begin{tabular}{|c|c|c|c|c|c|c|c|c|c|}
\hline \multirow[b]{2}{*}{ Compounds } & \multirow[b]{2}{*}{$\mathrm{KI}$} & \multirow[b]{2}{*}{ RT } & \multicolumn{3}{|c|}{ Ascorbate Addition (Asc) } & \multicolumn{2}{|c|}{ Black Pepper Level (BP) } & \multicolumn{2}{|c|}{$P$} \\
\hline & & & $\begin{array}{l}\text { Without } \\
\text { Asc }\end{array}$ & With Asc & $5 \mathrm{~g} / \mathrm{kg}$ & $10 \mathrm{~g} / \mathrm{kg}$ & $15 \mathrm{~g} / \mathrm{kg}$ & Asc & $\mathrm{BP}$ \\
\hline Methyl 2-propenyl disulfide & 969 & $\mathrm{C}$ & $3.46 \pm 1.74 \mathrm{a}$ & $2.30 \pm 1.15 b$ & $3.98 \pm 2.00 \mathrm{a}$ & $2.25 \pm 1.03 \mathrm{~b}$ & $2.43 \pm 0.92 \mathrm{~b}$ & $*$ & $*$ \\
\hline $\begin{array}{l}\text { Methyl trans-propenyl- } \\
\text { disulfide }\end{array}$ & 977 & $\mathrm{~B}$ & $0.46 \pm 0.24 \mathrm{a}$ & $0.48 \pm 0.23 \mathrm{a}$ & $0.63 \pm 0.22 \mathrm{a}$ & $0.36 \pm 0.26 \mathrm{~b}$ & $0.43 \pm 0.11 \mathrm{ab}$ & ns & ns \\
\hline Di-2-propenyl- disulfide & 1135 & $\mathrm{~B}$ & $10.28 \pm 4.1 \mathrm{a}$ & $7.22 \pm 2.29 \mathrm{~b}$ & $11.31 \pm 4.8 \mathrm{a}$ & $7.23 \pm 1.96 \mathrm{~b}$ & $7.69 \pm 2.11 \mathrm{~b}$ & $*$ & $*$ \\
\hline \multicolumn{10}{|l|}{ Terpenes } \\
\hline$\alpha$-Thujene & 944 & $\mathrm{~B}$ & $3.15 \pm 1.28 \mathrm{a}$ & $3.35 \pm 1.42 \mathrm{a}$ & $2.51 \pm 0.40 \mathrm{~b}$ & $2.50 \pm 1.14 \mathrm{~b}$ & $4.73 \pm 0.71 \mathrm{a}$ & ns & $* *$ \\
\hline$\alpha$-Pinene & 951 & $\mathrm{C}$ & $4.61 \pm 1.79 \mathrm{a}$ & $4.86 \pm 2.19 \mathrm{a}$ & $2.94 \pm 0.52 \mathrm{c}$ & $4.18 \pm 1.01 b$ & $7.08 \pm 1.04 \mathrm{a}$ & ns & $* *$ \\
\hline Camphene & 970 & $\mathrm{C}$ & $0.38 \pm 0.21 \mathrm{a}$ & $0.48 \pm 0.23 \mathrm{a}$ & $0.49 \pm 0.29 \mathrm{a}$ & $0.41 \pm 0.23 \mathrm{a}$ & $0.38 \pm 0.12 \mathrm{a}$ & ns & ns \\
\hline$\beta$-Pinene & 996 & $\mathrm{C}$ & $25.6 \pm 5.99 \mathrm{a}$ & $26.4 \pm 7.26 \mathrm{a}$ & $27.23 \pm 7.8 \mathrm{a}$ & $24.13 \pm 6.38 \mathrm{a}$ & $27.84 \pm 7.89 \mathrm{a}$ & ns & ns \\
\hline$\alpha$-Phellandrene & 1019 & $\mathrm{C}$ & $5.48 \pm 2.95 \mathrm{a}$ & $5.59 \pm 3.25 \mathrm{a}$ & $3.53 \pm 0.33 b$ & $4.08 \pm 2.56 b$ & $9.00 \pm 1.70 \mathrm{a}$ & ns & $* *$ \\
\hline 3-Carene & 1026 & $\mathrm{C}$ & $5.63 \pm 2.13 \mathrm{a}$ & $5.14 \pm 2.04 \mathrm{a}$ & $3.06 \pm 0.45 \mathrm{c}$ & $5.91 \pm 1.64 \mathrm{~b}$ & $7.18 \pm 0.91 \mathrm{a}$ & ns & $* *$ \\
\hline$\alpha$-Terpinene & 1042 & $\mathrm{C}$ & $2.33 \pm 0.65 \mathrm{a}$ & $2.50 \pm 0.71 \mathrm{a}$ & $2.16 \pm 0.48 \mathrm{~b}$ & $2.11 \pm 0.70 \mathrm{~b}$ & $2.97 \pm 0.46 \mathrm{a}$ & ns & $*$ \\
\hline D-Limonene & 1054 & A & $21.76 \pm 7.4 \mathrm{a}$ & $19.5 \pm 10.8 \mathrm{a}$ & $14.39 \pm 3.6 \mathrm{~b}$ & $16.92 \pm 5.35 b$ & $30.59 \pm 7.6 \mathrm{a}$ & ns & $* *$ \\
\hline$\beta$-Phellandrene & 1060 & $\mathrm{C}$ & $4.39 \pm 1.61 \mathrm{a}$ & $4.30 \pm 1.96 \mathrm{a}$ & $3.38 \pm 0.70 \mathrm{~b}$ & $3.43 \pm 1.34 \mathrm{~b}$ & $6.23 \pm 1.32 \mathrm{a}$ & ns & $* *$ \\
\hline Cis-Ocimene & 1097 & $\mathrm{C}$ & $2.42 \pm 0.74 \mathrm{a}$ & $2.54 \pm 0.59 \mathrm{a}$ & $2.75 \pm 0.89 \mathrm{a}$ & $2.24 \pm 0.39 \mathrm{a}$ & $2.47 \pm 0.56 \mathrm{a}$ & ns & ns \\
\hline$\gamma$-Terpinene & 1099 & $\mathrm{C}$ & $30.20 \pm 8.5 a$ & $28.16 \pm 5.2 \mathrm{a}$ & $33.2 \pm 7.59 \mathrm{a}$ & $26.36 \pm 6.14 a$ & $27.95 \pm 5.98 \mathrm{a}$ & ns & ns \\
\hline Terpinolene & 1104 & $\mathrm{C}$ & $1.77 \pm 0.43 \mathrm{a}$ & $1.17 \pm 0.42 \mathrm{a}$ & $1.11 \pm 0.55 \mathrm{a}$ & $1.02 \pm 0.34 \mathrm{a}$ & $1.38 \pm 0.25 \mathrm{a}$ & ns & ns \\
\hline Linalool & 1161 & A & $6.94 \pm 2.86 \mathrm{a}$ & $6.06 \pm 1.50 \mathrm{a}$ & $7.25 \pm 2.64 \mathrm{a}$ & $6.47 \pm 2.52 \mathrm{a}$ & $5.77 \pm 1.58 \mathrm{a}$ & ns & ns \\
\hline 4-Terpinenol & 1235 & B & $3.25 \pm 1.09 \mathrm{a}$ & $2.87 \pm 0.91 \mathrm{a}$ & $2.82 \pm 1.19 \mathrm{a}$ & $2.83 \pm 0.58 \mathrm{a}$ & $3.53 \pm 1.11 \mathrm{a}$ & ns & ns \\
\hline$\alpha$-Terpineol & 1258 & $\mathrm{C}$ & $2.44 \pm 1.51 \mathrm{a}$ & $2.48 \pm 0.81 \mathrm{a}$ & $2.54 \pm 0.65 \mathrm{a}$ & $2.68 \pm 1.95 \mathrm{a}$ & $2.16 \pm 0.46 \mathrm{a}$ & ns & ns \\
\hline Delta elemene & 1391 & $\mathrm{C}$ & $2.36 \pm 0.78 \mathrm{a}$ & $2.29 \pm 0.76 \mathrm{a}$ & $1.83 \pm 0.50 \mathrm{a}$ & $2.46 \pm 0.63 \mathrm{ab}$ & $2.69 \pm 0.88 \mathrm{~b}$ & ns & ns \\
\hline 2-caren-10-al & 1393 & $\mathrm{C}$ & $1.88 \pm 1.00 \mathrm{a}$ & $1.55 \pm 0.95 \mathrm{a}$ & $2.06 \pm 0.56 \mathrm{a}$ & $1.86 \pm 1.29 \mathrm{a}$ & $1.23 \pm 0.82 \mathrm{a}$ & ns & ns \\
\hline$\alpha$-Cubebene & 1396 & $\mathrm{C}$ & $0.87 \pm 0.57 \mathrm{a}$ & $0.88 \pm 0.28 \mathrm{a}$ & $0.80 \pm 0.18 \mathrm{a}$ & $0.76 \pm 0.56 \mathrm{a}$ & $1.05 \pm 0.48 \mathrm{a}$ & ns & ns \\
\hline Copaene & 1433 & $\mathrm{C}$ & $5.76 \pm 2.73 \mathrm{a}$ & $6.19 \pm 2.11 \mathrm{a}$ & $4.44 \pm 0.30 \mathrm{~b}$ & $5.27 \pm 2.85 b$ & $8.22 \pm 1.28 \mathrm{a}$ & ns & $* *$ \\
\hline Eugenol & 1456 & $\mathrm{~B}$ & $3.67 \pm 1.27 \mathrm{a}$ & $3.79 \pm 1.37 \mathrm{a}$ & $3.30 \pm 0.71 \mathrm{a}$ & $4.15 \pm 1.45 \mathrm{a}$ & $3.74 \pm 1.56 \mathrm{a}$ & ns & ns \\
\hline Trans-Caryophyllene & 1468 & $\mathrm{~B}$ & $2.02 \pm 0.73 \mathrm{a}$ & $1.64 \pm 0.64 \mathrm{a}$ & $1.56 \pm 0.39 \mathrm{~b}$ & $1.49 \pm 0.66 \mathrm{~b}$ & $2.43 \pm 0.64 \mathrm{a}$ & ns & ** \\
\hline$\alpha$-trans- Bergamotene & 1472 & $\mathrm{C}$ & $2.87 \pm 1.53 \mathrm{a}$ & $3.06 \pm 1.67 \mathrm{a}$ & $1.81 \pm 0.60 \mathrm{~b}$ & $2.77 \pm 1.75 b$ & $4.32 \pm 0.99 \mathrm{a}$ & ns & $* *$ \\
\hline Caryophyllene & 1490 & $\mathrm{~B}$ & $22.2 \pm 12.9 \mathrm{a}$ & $24.7 \pm 13.6 \mathrm{a}$ & $14.2 \pm 3.89 \mathrm{~b}$ & $20.7 \pm 14.84 b$ & $35.44 \pm 7.2 \mathrm{a}$ & ns & ** \\
\hline$\alpha$-Caryophyllene & 1504 & $\mathrm{~B}$ & $1.97 \pm 1.09 \mathrm{a}$ & $2.26 \pm 1.19 \mathrm{a}$ & $1.30 \pm 0.41 \mathrm{~b}$ & $1.99 \pm 1.30 \mathrm{~b}$ & $3.04 \pm 0.74 \mathrm{a}$ & ns & $* *$ \\
\hline
\end{tabular}

$P$ : statistical significance; ns: not significant $P>0.05 ; *: P<0.05 ; * *: P<0.01$. Results are expressed in Arbitrary Area Units $\left(\times 10^{6}\right)$; RI: Reliability of identification; A: mass spectrum and retention time identical with an authentic sample; B: mass spectrum and Kovats index from literature in accordance; C: tentative identification by mass spectrum; KI: Kovats index calculated for DB-624 capillary column $(60 \mathrm{~m} \times 0.25 \mathrm{~mm} \times 1.4 \mu \mathrm{m})$ installed on a gas chromatograph equipped with a mass selective detector. Different letters $(\mathrm{a}-\mathrm{c})$ indicate significant differences between the levels in the groups.

Another volatile chemical group identified in sucuk is esters. The esters determined were hexyl butyrate and methyl acetate. None of the compounds were affected by the use of ascorbate. The black pepper level of $15 \mathrm{~g} / \mathrm{kg}$ showed lower abundance for hexyl butyrate than for $5 \mathrm{~g} / \mathrm{kg}(P$
$>0.05$ ) (Table 2). Esters have low threshold values and give a fruity note to dry fermented sausages. The presence of alcohols and acids, as well as the esterification activity of staphylococci play an important role in ester formation (Xiao et al., 2020). 
Among the sulfur compounds, allyl mercaptan showed a higher abundance in sucuk samples. However, this compound was not influenced by the addition of ascorbate $(P>0.05)$. The use of ascorbate affected the levels of 3,3-thiobis-1propene, allyl methyl sulfide, methyl-2-propenyldisulfide, di-2-propenyl-disulfide significantly $(P$ $<0.05)$. The use of ascorbate in sucuk production reduced the abundance of some sulfur compounds (Table 2). Sulfur compounds can be formed by enzymatic reactions (allium types such as onion and garlic or vegetables such as broccoli, cabbage and cauliflower) or by heat treatment applications. Due to their low aroma and taste thresholds, volatile sulfur compounds can exhibit sensory potential at low concentrations (McGorrin, 2011). The black pepper level was also significantly effective on 1- (methylthio)propene, dimethyl disulfide, methyl 2-propenyldisulfide and di-2-propenyl-disulfide compounds $(P<0.05)$. Increasing the level of black pepper in sucuk production from $5 \mathrm{~g} / \mathrm{kg}$ to $10 \mathrm{~g} / \mathrm{kg}$ led to a decrease in the abundance of 1- (methylthio) - 1propene, dimethyl disulfide, methyl 2-propenyldisulfide and di-2-propenyl-disulfide compounds. However, increasing the black pepper level to 15 $\mathrm{g} / \mathrm{kg}$ caused no significant difference $(P>0.05)$.

The use of ascorbate had no significant effect on terpenes in sucuk samples $(P>0.05)$. On the other hand, the black pepper level was effective on 12 of 24 terpenes at the level of $P<0.05$ or $P<0.01$ (Table 2). Terpenes play an important role in the volatile profile of sucuk. In addition to black pepper, many spices such as garlic, red pepper, allspice and cumin are used in the sucuk (Kaban, 2010). The main source of terpenes are spices (Flores, 2018). It is reported that the main volatile compounds of black pepper are caryophyllene, Dlimonene, 3-carene (Li et al., 2020). In addition, volatile compounds such as alpha-pinene, sabinene, 1-alpha-pellandrene are included in the volatile profile of black pepper (Chi and $\mathrm{Wu}$, 2007; Lilie et al., 2007; Munekata et al., 2020). However, volatile profile of black pepper varies depending on the many factors such as chemotypes, geographic origin, seasonality, and use of oil extraction methods ( $\mathrm{Li}$ et al., 2020). In the present study, caryophyllene, D-limonene, and $\gamma$ terpinene were the most volatile compounds with the highest abundance among the terpenes in sucuk samples. Of these compounds, $\boldsymbol{\gamma}$-Terpinene was not affected by the black pepper level $(P$ $>0.05)$. On the other hand, caryophyllene and $\mathrm{D}$ limonene, which are of the main volatile compounds of black pepper, showed higher abundance at $15 \mathrm{~g} / \mathrm{kg}$ black pepper level (Table 2). 3-Carene, a bicyclic monoterpene, increased with increasing black pepper level. When the black pepper level was increased from $5 \mathrm{~g} / \mathrm{kg}$ to $15 \mathrm{~g} / \mathrm{kg}$, the abundance of this compound increased approximately 2.5 times (Table 2). $\alpha$ pinene also increased significantly as black pepper levels increased. In sucuk samples with $5 \mathrm{~g} / \mathrm{kg}$ black pepper and sucuk samples with $10 \mathrm{~g} / \mathrm{kg}$ black pepper, it was found that there were no significant differences in terms of $\alpha$-thujene, alpha-phellandrene, $\alpha$-terpinene, D-limonene, beta-phellandrene, copaene, trans-caryophyllene, alpha-trans-bergamotene, caryophyllene, alphacaryophyllene (Table 2). The black pepper content of $15 \mathrm{~g} / \mathrm{kg}$ showed a higher abundance for these compounds than for 5 and $10 \mathrm{~g} / \mathrm{kg}$.

\section{Principal Component Analysis (PCA)}

PCA was performed to evaluate the relationships between factors (ascorbate addition and black pepper level) and volatile compounds. The eigenvalues, variances and their cumulative proportions are shown in Table 3. The eigenvectors of the first three principal components (PC) are given in Table 4. The first three principal components (PC1, PC2 and PC3) were analyzed having eigenvalues greater than 1.0; explaining $78.40,17.90$ and $3.70 \%$ of the variance, respectively. The first two principal components explained $96.30 \%$ of the total variance (Table 3). It was found that D-limonene and caryophyllene were the most related variable for PC1; allyl mercaptane, $\gamma$-terpinene, $\mathrm{D}$-limonene, hexane for PC2 (Table 4). 
Table 3. Eigenvalue scores, variances and their cumulative proportions of first three components

\begin{tabular}{lccccc}
\hline \multirow{2}{*}{ Principal component } & \multicolumn{2}{c}{ Eigenvalue } & & \multicolumn{2}{c}{ Explained variance } \\
\cline { 2 - 3 } \cline { 5 - 6 } & For PC & Cumulative & & For PC & Cumulative \\
\hline PC1 & 119.00 & 119.00 & & 78.40 & 78.40 \\
PC2 & 27.23 & 146.23 & & 17.90 & 96.30 \\
PC3 & 5.57 & 151.80 & & 3.70 & 100.00 \\
\hline
\end{tabular}

Table 4. Eigenvectors of the variance-covariance matrix used for PCA

\begin{tabular}{|c|c|c|c|}
\hline Variable & PC1 & PC2 & PC3 \\
\hline Pentanal & -0.004 & 0.014 & 0.023 \\
\hline Hexanal & -0.036 & 0.039 & -0.003 \\
\hline Octanal & -0.025 & 0.011 & 0.129 \\
\hline Nonanal & -0.093 & 0.066 & 0.491 \\
\hline 2-methyl-3-phenyl-propanal & -0.116 & -0.020 & 0.159 \\
\hline Hexane & -0.087 & 0.406 & -0.578 \\
\hline Undecane & -0.007 & 0.001 & 0.014 \\
\hline Dodecane & -0.004 & 0.003 & -0.101 \\
\hline Tetradecane & -0.012 & 0.011 & -0.093 \\
\hline Ethanol & 0.018 & 0.033 & -0.029 \\
\hline Styrene & 0.004 & 0.002 & 0.003 \\
\hline 1,2-dimethoxy-4-(2-propenyl)-benzene & 0.018 & -0.067 & 0.010 \\
\hline Acetic acid & -0.018 & 0.092 & 0.233 \\
\hline Methyl acetate & -0.010 & 0.028 & -0.008 \\
\hline Hexyl butyrate & -0.030 & 0.007 & -0.058 \\
\hline Allyl mercaptan & -0.056 & 0.578 & 0.096 \\
\hline Allyl methyl sulfide & -0.044 & 0.157 & -0.068 \\
\hline 1-(methylthio) -1-propene & -0.004 & 0.016 & 0.030 \\
\hline Dimethyl disulfide & -0.015 & 0.028 & 0.002 \\
\hline 3,3-thiobis-1-Propene & -0.042 & 0.116 & -0.117 \\
\hline Methyl 2-propenyl-disulfide & -0.044 & 0.120 & 0.002 \\
\hline Methyl trans-propenyl-disulfide & -0.005 & 0.011 & 0.025 \\
\hline Di-2-propenyl-disulfide & -0.104 & 0.295 & -0.029 \\
\hline$\alpha$-thujene & 0.079 & 0.042 & 0.080 \\
\hline$\alpha$-pinene & 0.138 & 0.006 & 0.005 \\
\hline Camphene & -0.003 & -0.002 & 0.017 \\
\hline B-pinene & 0.042 & 0.269 & 0.149 \\
\hline$\alpha$-phellandrene & 0.190 & 0.084 & 0.093 \\
\hline 3-Carene & 0.123 & -0.060 & -0.247 \\
\hline$\alpha$-Terpinene & 0.029 & 0.014 & 0.046 \\
\hline D-Limonene & 0.551 & 0.302 & -0.116 \\
\hline$\beta$-phellandrene & 0.100 & 0.064 & 0.057 \\
\hline Cis-ocimene & -0.006 & 0.020 & 0.058 \\
\hline$\gamma$-Terpinene & -0.139 & 0.385 & 0.336 \\
\hline Terpinolene & 0.010 & 0.012 & 0.016 \\
\hline Linalool & -0.048 & 0.052 & -0.060 \\
\hline 4-Terpinenol & 0.025 & 0.032 & -0.029 \\
\hline$\alpha$-Terpineol & -0.015 & -0.019 & -0.020 \\
\hline
\end{tabular}


Table 2. continue

\begin{tabular}{lccc}
\hline Variable & PC1 & PC2 & PC3 \\
\hline Delta elemene & 0.026 & -0.016 & -0.050 \\
2-caren-10-al & -0.028 & 0.008 & -0.040 \\
$\alpha$ - cubebene & 0.009 & 0.008 & 0.013 \\
Copaene & 0.129 & 0.015 & 0.060 \\
Eugenol & 0.009 & -0.048 & -0.062 \\
Trans-caryophyllene & 0.030 & 0.040 & -0.017 \\
$\alpha$-Trans-bergamotene & 0.082 & -0.011 & -0.012 \\
Caryophyllene & 0.709 & -0.026 & 0.153 \\
$\alpha$ caryophyllene & 0.057 & -0.016 & 0.006 \\
\hline
\end{tabular}

PC1 was positively related with sucuk containing $15 \mathrm{~g} / \mathrm{kg}$ black pepper and with ascorbate. In addition, PC1 was inversely related with the levels of $5 \mathrm{~g} / \mathrm{kg}$ black pepper and $10 \mathrm{~g} / \mathrm{kg}$ black pepper as well as without ascorbate (Fig. 1). At the same time, PC1 separated the groups with ascorbate and without ascorbate well. On the other hand, sucuk samples with $5 \mathrm{~g} / \mathrm{kg}$ black pepper, $15 \mathrm{~g} / \mathrm{kg}$ black pepper and sucuk samples without ascorbate placed on positive side of PC2. PC2 showed that $10 \mathrm{~g} / \mathrm{kg}$ black pepper correlated more strongly with ascorbate than other black pepper levels, and placed on negative side for PC2 (Fig. 1).

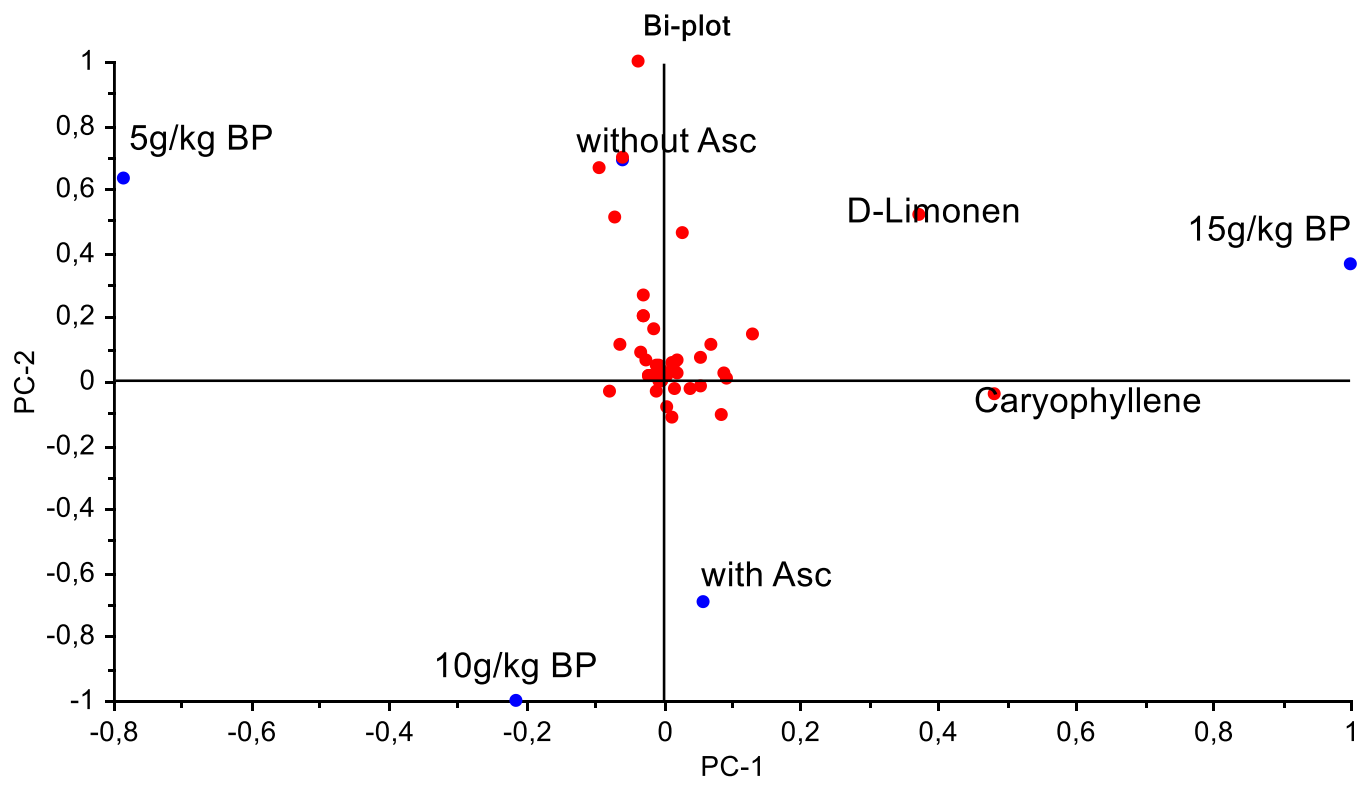

Figure 1: Principal component analysis biplot of the relationships between factors (sodium ascorbate addition and black pepper level) and volatile compounds

\section{CONCLUSION}

It is a notable finding from the study that ascorbate has no effect on aldehydes, which are mainly formed by lipid oxidation. Increasing the level of black pepper also resulted a decrease in the abundance of sulfur compounds. While the black pepper levels reduced hexanal levels, which is an indicator of lipid oxidation, it raised ethanol levels. Terpenes were the main group, in which black pepper is effective. An increase in the abundance of many terpene compounds has been observed with increasing black pepper level. The group with $15 \mathrm{~g} / \mathrm{kg}$ black pepper placed on the positive side of PC1, while the groups with 5 and 
$10 \mathrm{~g} / \mathrm{kg}$ black pepper placed on the negative side of PC1. The sucuk samples containing $15 \mathrm{~g} / \mathrm{kg}$ black pepper had a high relationship with Dlimonene and caryophyllene. In addition, some volatile compounds were affected by black pepper levels and showed different dimensions. Moreover, the use of ascorbate also affected the dimensions of some volatile compounds.

\section{ACKNOWLEDGMENTS}

The authors would also like to thank Atatürk University Eastern Anatolia High Technology Application and Research Center (DAYTAM) Directorate.

\section{DECLARATION OF COMPETING INTEREST}

The authors have declared no conflict of interest.

\section{AUTHORS' CONTRIBUTIONS}

Selen SALLAN performed investigation, formal analysis and writing-original draft. Güzin KABAN did methodology, validation and writing. Mükerrem KAYA was supervisior and administrated the project, performed writing review \& editing.

\section{REFERENCES}

Chi, S-P., Wu, Y-C. (2007). Spices and Seasonings, Editor: Fidel Toldra, Handbook of Fermented Meat and Poultry, pp. 87-100, Blackwell Publishing, USA

Demirok Soncu, E., Özdemir, N., Arslan, B., Küçükkaya, S., Soyer, A. (2020). Contribution of surface application of chitosan-thyme and chitosan-rosemary essential oils to the volatile composition, microbial profile, and physicochemical and sensory quality of dryfermented sausages during storage. Meat Sci, 166, 108127. doi:10.1016/j.meatsci.2020.108127

Ekici, L., Ozturk, I., Karaman, S., Caliskan, O., Tornuk, F., Sagdic, O., Yetim, H. (2015). Effects of black carrot concentrate on some physicochemical, textural, bioactive, aroma and sensory properties of sucuk, a traditional Turkish dry-fermented sausage. LWT- Food Sci Technol, 62(1), 2, 718726. doi:10.1016/j.lwt.2014.12.025
Flores, M. (2018). Understanding the implications of current health trends on the aroma of wet and dry cured meat products. Meat Sci, 144, 53-61. doi:10.1016/j.meatsci.2018.04.016

Huynh, D. B. T., Krickmeier, J., Schnaeckel, W. (2020). Effects of treatment temperatures on redox potential and sensory evaluation of different spices and herbs applied to cooked pork sausages. J Sci Food Agr, 100, 7, 2898-2904. doi:10.1002/jsfa.10316

Kaban, G. (2009). Changes in composition of volatile compounds and in microbiological and physicochemical parameters during pastırma processing. Meat Sci, 82, 1, 17-23. doi:10.1016/j.meatsci.2008.11.017

Kaban, G. (2010). Volatile compounds of traditional turkısh dry fermented sausage (Sucuk). Int J Food Prop, 13, 3, 525-534. doi:10.1080/10942910802688184

Kaban, G., Kaya, M. (2009). Effects of Lactobacillus plantarum and Staphylococcus xylosus on the quality characteristics of dry fermented sausage "sucuk". J Food Sci, 74(1), S58-S63. doi:10.1111/j.1750-3841.2008.01014.x

Kaya, M., Kaban, M. (2019). Fermente et ürünleri. Grda Biyoteknolojisi. Editör Necla ARAN, ss. 157195, 5. Basım, ISBN: 978-605-133-134-8, Nobel Yayınc1lik, İstanbul.

Li, Y-X., Zhanga, C., Shenyuan, P., Chena, L., Liua, M., Yanga, K., Zengc, X., Tiana, J. (2020). Analysis of chemical components and biological activities of essential oils from black and white pepper (Piper nigrum L.) in five provinces of southern China. LWT, Food Sci Technol, 117, 108644. doi:10.1016/j.lwt.2019.108644

Lilie, M., Hein, S., Wilhelm, P., Mueller, U. (2007). Decontamination of spices by combining mechanical and thermal effects - an alternative approach for quality retention. Int J Food Sci Technol, 42, 2, 190-193. Doi:10.1111/j.13652621.2006.01204.x

Mateo, J., Zumalacarregui, J.M. (1996). Volatile compounds in chorizo and their changes during ripening. Meat Sci, 44, 4, 255-273. doi:10.1016/S0309-1740(96)00028-9 
McGorrin, R. J. (2011). The significance of volatile sulfur compounds in food flavors.In: (M. Qian, X. Fan, K. Mahattanatawee (Eds), Volatile sulfur compounds in food, (pp.3-31). ACS Symposium Series 1068, ACS Books.

Montel, M.C., Mason, F. Talon, R. (1998). Bacterial Role in Flavour Development. Meat Sci, 49 (1), 111-123. doi:10.1016/S03091740(98)90042-0

Munekata, P. E. S., Rocchetti, G., Pateiro, M., Lucini, L., Dominguez, R., Lorenzo, J M. (2020). Addition of plant extracts to meat and meat products to extend shelf-life and healthpromoting attributes: an overview. Curr Opin Food Sci, 31:81-87. doi:10.1016/j.cofs.2020.03.003

Ordonez, J. A., Hierro, E. M., Bruna, J. M., de la Hoz, L. (1999). Changes in the components of dry-fermented sausages during ripening. Crit Rev Food Sci Nutr, 39, 4, 329-367. doi:10.1080/10408699991279204

Ramirez, R., Cava, R. (2007). Volatile Profiles of Dry -Cured Meat Products From Three Different Iberian x duroc Genotypes. J Agric Food Chem, 55: 1923-1931. doi:10.1021/jf0628101

Ruiz, J., Ventanas, J., Cava, R., Andres, A., Garcia, C. (1999). Volatile Compounds of Dry-Cured Iberian Ham as Affected by the Length of the Curing Process. Meat Sci, 52, 1, 19- 27. doi:10.1016/S0309-1740(98)00144-2

Sallan, S., Kaban, G., Kaya, M. (2019). Nitrosamines in Sucuk: Effects of black pepper, sodium ascorbate and cooking level. Food Chem, 288,341-346. doi:10.1016/j.foodchem. 2019.02.129

Sallan, S., Kaban, G., Kaya, M. (2022). The effects of nitrite, sodium ascorbate and starter culture on volatile compounds of a semi-dry fermented sausage. LWT, Food Sci Technol, 153, 112540.

Sidira, M., Kandylis, P., Kanellaki, M., Kourkoutas, Y. (2015). Effect of immobilized Lactobacillus casei on volatile compounds of heat treated probiotic dry-fermented sausages. Food
Chem, 178, 201-207. doi: 10.1016/j.foodchem.2015.01.068

Srinivasan, K. (2007). Black Pepper and its Pungent Principle-Piperine: A Review of Diverse Physiological Effects. Crit Rev Food Sci Nutr, 47, 735-748. doi:10.1080/10408390601062054

Stahnke, L. H. (1999). Volatiles produced by Staphylococcus xylosus and Staphylococcus carnosus during growth in sausage minces Part II. The influence of growth parameters. LWT-Food Sci and Technol, 32, 365-371. doi:10.1006/fstl.1999.0559

Toldra, F. (1998). Proteolysis and Lipolysis in Flavour Development of Dry-cured Meat Products. Meat Sci, 49, 1, S101-S110. doi:10.1016/S0309-1740(98)90041-9

Vignolo, G., Fontana, C., Fadda, S. (2010). Semidry and Dry Fermented Sausages, Handbook of Meat Processing, Editor: Fidel Toldra, Online ISBN: 9780813820897

Xiao, Y., Liu, Y., Chen, C., Xie, T., Li, P. (2020). Effect of Lactobacillus plantarum and Staphylococcus xylosus on flavour development and bacterial communities in Chinese dry fermented sausages. Food Res Int, 135, 109247. doi:10.1016/ j.foodres.2020.109247

Yalınkılıç, B., Kaban, G., Ertekin, Ö., Kaya, M. (2015). Determination of volatile compounds of sucuk with different orange fiber and fat levels. Kafkas Unv Vet Fak Dergisi, 21(2), 233-239. doi:10.9775/kvfd.2014.12197

Zarai, Z., Boujelbene, E., Salem, N. B., Gargouri, Y., Sayari, A. (2013). Antioxidant and antimicrobial activities of various solvent extracts, piperine and piperic acid from Piper nigrum. LWT-Food Sci Technol, 50, 2, 634-641. doi:10.1016/j.lwt.2012.07.036

Zhang, C. Zhao, J., Famous, E., Pan, S., Peng, X., Tian, J. (2021). Antioxidant, hepatoprotective and antifungal activities of black pepper (Piper nigrum L.) essential oil. Food Chem, 346, 128845. doi:10.1016/j.foodchem.2020.128845 